University of Texas at El Paso

ScholarWorks@UTEP

$6-2014$

\title{
How to Estimate Relative Spatial Resolution of Different Maps or Images of the Same Area?
}

Christian Servin

El Paso Community College, cservin@gmail.com

\section{A A. Velasco}

The University of Texas at El Paso, velasco@geo.utep.edu

Vladik Kreinovich

The University of Texas at El Paso, vladik@utep.edu

Follow this and additional works at: https://scholarworks.utep.edu/cs_techrep

Part of the Computer Sciences Commons, and the Geography Commons

Comments:

Technical Report: UTEP-CS-14-34a

Published in Proceedings of IEEE International Conference on Systems, Man, and Cybernetics SMC'2014, San Diego, California, October 5-8, 2014, pp. 3507-3511.

\section{Recommended Citation}

Servin, Christian; Velasco, A A.; and Kreinovich, Vladik, "How to Estimate Relative Spatial Resolution of Different Maps or Images of the Same Area?" (2014). Departmental Technical Reports (CS). 855.

https://scholarworks.utep.edu/cs_techrep/855

This Article is brought to you for free and open access by the Computer Science at ScholarWorks@UTEP. It has been accepted for inclusion in Departmental Technical Reports (CS) by an authorized administrator of ScholarWorks@UTEP.For more information, please contact Iweber@utep.edu. 


\section{How to Estimate Relative Spatial Resolution of Different Maps or Images of the Same Area?}

\author{
Christian Servin ${ }^{1,2}$ \\ ${ }^{1}$ Information Technology Dept. \\ El Paso Community College \\ 919 Hunter, El Paso, Texas 79915, USA \\ Email: cservin@gmail.com
}

\author{
Aaron Velasco ${ }^{3,4}$ \\ ${ }^{3}$ Dept. of Geological Sciences \\ ${ }^{4}$ Cyber-ShARE Center \\ University of Texas at El Paso \\ El Paso, Texas 79968, USA \\ Email: aavelasco@utep.edu
}

\author{
Vladik Kreinovich ${ }^{2,4}$ \\ ${ }^{2}$ TRACS Center, Dept. of Computer Science \\ ${ }^{4}$ Cyber-ShARE Center \\ University of Texas at El Paso \\ El Paso, Texas 79968, USA \\ Email:vladik@utep.edu
}

\begin{abstract}
In this paper, we describe how to estimate relative spatial resolution of different maps or images of the same area under uncertainty. We consider probabilistic and fuzzy approaches and we show that both approaches lead to the same estimate - which makes us somewhat more confident that this joint result is reasonable.
\end{abstract}

\section{INTRODUCTION}

Different measurements results in maps of different spatial resolution. In many practical situations, we have several maps or images of the same areas, with different accuracy and different spatial resolution.

Example: geosciences. For example, in geosciences, we have, among others, two different types of data: gravity data and seismic data.

Gravity measurements measure the exact gravity force at different locations. These measurements enable us to distinguish areas where the material is heavier - and thus, causes more gravitational field - from the areas where material is lighter. Based on measurements of each type, we can develop a 3-D map describing the densities at different spatial locations and at different depths.

We can measure the gravity force at each location with a very high accuracy; as a result, each gravity measurement is very accurate. However, each gravity measurement describes not just the density at this particular location, but the overall effect of the densities at all neighboring areas as well. By using only gravity measurements, it is very difficult to distinguish between two nearby spatial locations. As a result, the gravitybased map is accurate, but it has a low spatial resolution.

Seismic data consists of the arrival times of a seismic signal from a natural earthquake - or of an artificial small explosion set up for the whole purpose of seismic analysis. Each travel time reflects the path by which a seismic wave propagated from the source to the sensor. This path is narrow, so the resulting map has a high spatial resolution - but its accuracy is much lower, since the small seismic signal has to be detected against a noisy seismic background.

Need to fuse the corresponding maps. The above example from geophysics describes a typical situation when we have two different maps describing the same area:
- $\quad$ one of the maps has very accurate values, but with a low spatial resolution, while

- another map has higher spatial resolution but much less accurate values.

In other words, the second map provides approximate values of the quantity at different 3-D locations, while the first maps provides very accurate values of the quantity averaged over some subareas.

It is desirable to combine all this information into a single 3-D map.

To fuse the maps, we need to know their relative spatial resolution. If we know how exactly the two maps are related to the actual quantity, we can then apply the Least Squares method to the two corresponding equations and find the map which is the best fit for both types of data; see, e.g., [6], [7], [8], [9], [10], [11]. The problem is that in many cases - and geophysics is one of these cases - we do not know how exactly these maps are related to the actual (unknown) spatial distribution of the corresponding quantity. In other words, we do not know the relative spatial resolution of the two maps, we need to find this resolution based on the maps themselves.

What we do in this paper. In this paper, we use fuzzy and probabilistic methods to come up with algorithms for determining relative spatial resolution of two maps. Interestingly, both techniques lead to similar estimate - which makes us somewhat more confident about the validity of this common approach.

\section{What Is the Relation Between IMAGeS With DifFERENT Spatial Resolution: Probabilistic AND FUZZY APPROACHES}

Need to perform estimations under uncertainty. In order to fuse images $\widetilde{I}_{i}(x), i=1,2, \ldots$ corresponding to different spatial resolution, we need to find out how the observed images $\widetilde{I}_{i}(x)$ are related to the actual (ideal) image $I(x)$.

As we have mentioned, we consider a practically important case of uncertainty, when we do not have an exact formula describing the relation between the observed images $\widetilde{I}_{i}(x)$ and 
the actual image, and we need to extract this information from the observed images themselves.

Possible approaches to estimation under uncertainty. Traditionally, uncertainty in science and engineering is handled by the probabilistic approach. This approach originated in situations when we can experimentally determine the frequencies (probabilities) of different image distortions, but it is also actively used in situations when we only have partial information about these probabilities - or even, as in our case, we do not have any information about these probabilities at all. In such situations, we can use some commonsense principles to come up with the missing probabilities; see, e.g., [1], [12].

For example, if we have two alternatives and we have no reason to assume that one of the them is more frequent than the other one, then it makes sense to assume that these two alternatives have equal probabilities - which are thus equal to $1 / 2$. If we have $n$ alternatives, and we have no information about which alternatives are more probable and which are less probable, it makes sense to assume that all these $n$ alternatives are equally probable.

Similarly, if we only know that a quantity $x$ is located on an interval $[0,1]$, and we do not know which values from this interval are more probable or less probable, it makes sense to assume that all these possible values are equally probable, i.e., that we have a uniform probability distribution on the interval $[0,1]$.

Instead of "making up" the unknown probabilities based on common sense, another idea is to explicitly formalize the commonsense ideas. This formalization is usually not easy, because our commonsense ideas are often formulated in terms of imprecise ("fuzzy") words from natural language. For this formalization, we can use fuzzy techniques, techniques specifically developed to formalize natural-language knowledge; see, e.g., [2], [4], [13].

What we do in this section. In this section, to find the relation between $\widetilde{I}_{i}(x)$ and $I(x)$, we use both probabilistic and fuzzy approaches. Interestingly, we get the same relation in both cases. The fact that the same relation comes from two completely different set of ideas makes us somewhat more confident that this is indeed the right relation between the observed and actual images.

Because of this increased confidence, in the following section, we use this relation to determine the relative spatial resolution of two maps.

Relation between $\widetilde{I}_{i}(x)$ and $I(x)$ : probabilistic approach. Spatial uncertainty means that the value which is actually located at a point $x$ is observed as corresponding to a slightly different point $\widetilde{x}=x+\Delta x$. As a result, each value $I(x)$ gets distributed to values $I(x+\Delta x)$, for the corresponding random variable $\Delta x$.

In general, there are many independent sources of spatial uncertainty. The resulting spatial deviations $\Delta x$ can therefore be represented as a sum of many small independent random variables. It is known that under reasonable assumptions, the distribution of such sums is close to Gaussian; this is known as the Central Limit Theorem; see, e.g., [12]. We can therefore conclude that $\Delta x$ is normally distributed. In the isotropic case, this distribution has the probability density

$$
\rho(\Delta x)=\frac{1}{2 \pi \cdot \sigma} \cdot \exp \left(-\frac{\|\Delta x\|^{2}}{2 \sigma^{2}}\right) .
$$

Each original value $I(x)$ is thus distributed, with this density, among the neighboring values, contributing a proportional value $I(x) \cdot \rho(\Delta x) d \Delta x$ to each point $x+\Delta x$. The resulting observed overall intensity $\widetilde{I}(y)$ at a point $x$ can thus be obtained by adding up the values $I(x) \cdot \rho(\Delta x) d \Delta x$ corresponding to $x+\Delta x=y$ :

$$
\begin{gathered}
\tilde{I}(y)=\int I(x) \cdot f(y-x) d x= \\
\text { const } \cdot \int I(x) \cdot \exp \left(-\frac{\|y-x\|^{2}}{2 \sigma^{2}}\right) d x ;
\end{gathered}
$$

see, e.g., [5]

Relation between $\widetilde{I}(x)$ and $I(x)$ : fuzzy approach. In fuzzy approach, instead of trying to guess the corresponding probabilities, we explicitly formalize the corresponding commonsense knowledge. The corresponding rules for each observed value $\widetilde{I}(y)$ are straightforward: the value of $\widetilde{I}(y)$ comes from the values $I(x)$ for points $x$ which are close to $y$. In more precise terms, we have the following rules:

$$
\text { If } x \text { is close to } y \text {, then } \widetilde{I}(y) \text { is close to } I(x) \text {. }
$$

In [3], we have shown that, under reasonable assumptions, a natural way to describe closeness is by using a Gaussian membership function, which, in the isotropic case, has the form

$$
\mu(\Delta x)=\exp \left(-\frac{\|\Delta x\|^{2}}{2 \sigma^{2}}\right),
$$

where $\Delta x \stackrel{\text { def }}{=} y-x$.

For reach $y$, by combining the values coming from different rules, we conclude that, for each $x$, the value $\widetilde{I}(y)$ is equal to $I(x)$ with degree of membership $\mu(y-x)$. In other words, we have a membership function that attains values $I(x)$ with degree of membership $\mu(y-x)$. We want to transform this fuzzy information into a single (crisp) value, i.e., to defuzzify the original membership function. The experience of fuzzy control [2], [4] has shown that in most cases, the most effective defuzzification procedure is centroid defuzzification, when we transform the original membership function $m(t)$ into the crisp value

$$
\bar{t}=\frac{\int t \cdot m(t) d t}{\int m(t) d t} .
$$

In our case, we get the value

$$
\widetilde{I}(y)=\frac{\int I(x) \cdot \mu(y-x) d x}{\int \mu(y-x) d x} .
$$

The denominator is a constant not depending on $y$. Substituting the expression for the Gaussian membership function into this formula, we conclude that

$$
\widetilde{I}(y)=\text { const } \cdot \int I(x) \cdot \exp \left(-\frac{\|y-x\|^{2}}{2 \sigma^{2}}\right) d x .
$$


Relation between $\widetilde{I}(x)$ and $I(x)$ : conclusion. From the previous two subsections, we can see that both approaches lead to the exact same relation between the observed image $\widetilde{I}(x)$ and the actual image $I(x)$ : namely, that $\widetilde{I}(x)$ is proportional to the result of a convolution between the original image $I(x)$ and the Gaussian kernel.

This coincidence is a good indication that this is probably an adequate model for spatial distortion.

\section{How to Estimate Relative Spatial RESOLUTION?}

Analysis of the problem. Let us use the above model to come up with an algorithm for estimating relative spatial resolution between two images or maps.

First approximation. Specifically, we have two images $\widetilde{I}_{1}(x)$ and $\widetilde{I}_{2}(x)$. According to the above formulas describing spatial distortion, we have

$$
\widetilde{I}_{1}(y)=C_{1} \cdot \int I(x) \cdot \exp \left(-\frac{\|y-x\|^{2}}{2 \sigma_{1}^{2}}\right) d x
$$

and

$$
\widetilde{I}_{2}(y)=C_{2} \cdot \int I(x) \cdot \exp \left(-\frac{\|y-x\|^{2}}{2 \sigma_{2}^{2}}\right) d x .
$$

We are interested in the values $\sigma_{1}$ and $\sigma_{2}$ which characterize the spatial resolution corresponding to each image or map.

What can we determine about the spatial resolutions $\sigma_{1}$ and $\sigma_{2}$ ? Can we determine both spatial resolutions $\sigma_{1}$ and $\sigma_{2}$ ? If not, what can we determine about the values $\sigma_{i}$ ?

To answer these questions, it is useful to take into account that formulas involving convolution are greatly simplified if we use Fourier transform: namely, the Fourier transform $H(\omega)$ of the convolution $h(y)=\int f(y) \cdot g(x-y) d x$ is equal to the product $H(\omega)=F(\omega) \cdot G(\omega)$ of the Fourier transforms of the functions $f(x)$ and $g(x)$. The Fourier transform $G(\omega)$ of the Gaussian function

$$
g(x) \stackrel{\text { def }}{=} \exp \left(-\frac{\|x\|^{2}}{2 \sigma^{2}}\right)
$$

is known to also be a Gaussian:

$$
G(\omega)=\exp \left(-\frac{1}{2} \cdot\|\omega\|^{2} \cdot \sigma^{2}\right)
$$

Thus, in this first approximation, for Fourier transforms $F_{1}(\omega)$ and $F_{2}(\omega)$ of the observed images, we get

$$
\begin{aligned}
& F_{1}(\omega)=C_{1} \cdot F(\omega) \cdot \exp \left(-\frac{1}{2} \cdot\|\omega\|^{2} \cdot \sigma_{1}^{2}\right) ; \\
& F_{2}(\omega)=C_{2} \cdot F(\omega) \cdot \exp \left(-\frac{1}{2} \cdot\|\omega\|^{2} \cdot \sigma_{2}^{2}\right),
\end{aligned}
$$

where $F(\omega)$ is the Fourier transform of the original image.

From the above equations, one can conclude that

$$
F_{2}(\omega)=C \cdot F_{1}(\omega) \cdot \exp \left(-\frac{1}{2} \cdot\|\omega\|^{2} \cdot\left(\sigma_{2}^{2}-\sigma_{1}^{2}\right)\right) .
$$

Thus, when we only know two different maps, the only information that we can extract from these maps is the difference

$$
\Delta \stackrel{\text { def }}{=} \sigma_{2}^{2}-\sigma_{1}^{2} \text {. }
$$

It is reasonable to call this difference relative spatial resolution of the two images (maps), since it describes the difference between spatial resolutions of different images.

A more realistic description. The above first-approximation description takes into account spatial distortion, but it idealizes the situation by assuming that only the spatial location changes while the actual value of the quantity is measured exactly. Of course, the actual value is also not measured exactly, there is always a difference $\Delta q \stackrel{\text { def }}{=} \widetilde{q}-q$ between the measured value $\widetilde{q}$ and the actual value $q$. In other words, the measured value $\widetilde{q}$ is not exactly equal to $q$, it is equal to $q+\Delta q$, for some $\Delta q$. This measurement error is added to the actual (unknown) value and is, therefore, known as the additive noise.

If we take additive noise $n_{i}(y)$ into account, then we get more realistic formulas for describing the dependence between the actual and observed images:

$$
\begin{aligned}
& \widetilde{I}_{1}(y)=C_{1} \cdot \int I(x) \cdot \exp \left(-\frac{\|y-x\|^{2}}{2 \sigma_{1}^{2}}\right) d x+n_{1}(y) \\
& \widetilde{I}_{2}(y)=C_{2} \cdot \int I(x) \cdot \exp \left(-\frac{\|y-x\|^{2}}{2 \sigma_{2}^{2}}\right) d x+n_{2}(y) .
\end{aligned}
$$

As a result, for Fourier transforms, we get

$$
\begin{aligned}
& F_{1}(\omega)=C_{1} \cdot F(\omega) \cdot \exp \left(-\frac{1}{2} \cdot\|\omega\|^{2} \cdot \sigma_{1}^{2}\right)+N_{1}(\omega) ; \\
& F_{2}(\omega)=C_{2} \cdot F(\omega) \cdot \exp \left(-\frac{1}{2} \cdot\|\omega\|^{2} \cdot \sigma_{2}^{2}\right)+N_{2}(\omega),
\end{aligned}
$$

where $N_{i}(\omega)$ denotes the Fourier transform of the additive noises $n_{1}(y)$ and $n_{2}(y)$. From these equations, we can conclude that

$$
F_{2}(\omega)=C \cdot F_{1}(\omega) \cdot \exp \left(-\frac{1}{2} \cdot\|\omega\|^{2} \cdot\left(\sigma_{2}^{2}-\sigma_{1}^{2}\right)\right)+N(\omega),
$$

where

$$
C \stackrel{\text { def }}{=} \frac{C_{1}}{C_{2}}
$$

and

$$
N(\omega) \stackrel{\text { def }}{=} N_{2}(\omega)-N_{1}(\omega) \cdot \exp \left(-\frac{1}{2} \cdot\|\omega\|^{2} \cdot \Delta\right) .
$$

This is a model that we will use to reconstruct the relative spatial resolution $\Delta$.

How to find $C$. The coefficient $C$ can be found, e.g., by comparing the overall energy of two images - i.e., by comparing the values corresponding to $\omega=0$. For this value, the above formula takes the form

$$
F_{2}(0)=C \cdot F_{1}(0)+N(0) .
$$

Thus, we can estimate $C$ as

$$
C \approx \frac{F_{2}(0)}{F_{1}(0)} .
$$


Once the constant $C$ is estimated, we can divide the image $\widetilde{I}(x)$ (and thus, its Fourier transform $F_{2}(\omega)$ ) by this constant, and get a simpler relation between $F_{1}(\omega)$ and $F_{2}(\omega)$ :

$$
F_{2}(\omega)=F_{1}(\omega) \cdot \exp \left(-\frac{1}{2} \cdot\|\omega\|^{2} \cdot \Delta\right)+N(\omega) .
$$

What we know about the additive noise. In many practical cases, we do not know the exact characteristics of the additive noise, we only know its order of magnitude, i.e., we only know a number $n$ such that that $N(\omega) \approx n$.

We must limit ourselves to above-noise values. For frequencies $\omega$ for which $N_{2}(\omega) \approx n$, the whole observed value may be caused by noise. As a result, the corresponding values $N_{i}(\omega)$ do not carry any information about the actual image - and thus, do not carry any information about $\Delta$. Therefore, we must limit ourselves only to frequencies for which the signal is above noise, i.e., for which $\left|F_{2}(\omega)\right| \gg n$, i.e., for which $\left|F_{2}(\omega)\right| \geq c \cdot n$ for some constant $c \gg 1$.

For these frequencies, we have

$$
F_{2}(\omega) \approx F_{1}(\omega) \cdot \exp \left(-\frac{1}{2} \cdot\|\omega\|^{2} \cdot \Delta\right) \text { with accuracy } \approx n \text {. }
$$

It is sufficient to consider absolute values. The values of the Fourier transform are, in general, complex numbers. A complex number $z$ can be characterized by its absolute value (modulus) $|z|$ and its phase. As one can see from the above equation, the unknown $\Delta$ does not affect the phases, only absolute values, so it is sufficient to consider the absolute values of both sides:

$$
F_{2}(\omega) \approx F_{1}(\omega) \cdot \exp \left(-\frac{1}{2} \cdot\|\omega\|^{2} \cdot \Delta\right) \text { with accuracy } \approx n \text {. }
$$

From non-linear regression to linear regression. The above formula non-linearly depends on the unknown $\Delta$. To simplify the formula, it is desirable to reduce this dependence to a linear one. This can be done if we take logarithms of both sides, i.e., if instead of the absolute values $\left|F_{i}(\omega)\right|$ of the Fourier transforms, we consider their logarithms $\ell_{i}(\omega) \stackrel{\text { def }}{=} \ln \left(\left|F_{i}(\omega)\right|\right)$.

In taking the logarithms, we need to take into account that for a signal $q$ with noise $\Delta q \ll q$, we have

$$
\ln (q+\Delta q) \approx \ln (q)+\frac{\Delta q}{q} .
$$

Thus, if the original noise $\Delta q$ was of order $n$, the noise

$$
\frac{\Delta q}{q}
$$

of the logarithm is of order

$$
\frac{n}{q}
$$

Thus, after taking logarithms, we get

$$
\ell_{2}(\omega) \approx \ell_{1}(\omega)-\frac{1}{2} \cdot\|\omega\|^{2} \cdot \Delta \text { with accuracy } \approx \frac{n}{\left|F_{2}(\omega)\right|} .
$$

By moving the terms containing the unknown $\Delta$ to one side of the equation and all other terms to the other side and by dividing by the coefficient

$$
\frac{1}{2} \cdot\|\omega\|^{2}
$$

at $\Delta$, we conclude that

$$
\Delta \approx \frac{2\left(\ell_{1}(\omega)-\ell_{2}(\omega)\right)}{\|\omega\|^{2}} \text { with accuracy } \approx \frac{2 n}{\left|F_{2}(\omega)\right| \cdot\|\omega\|^{2}} .
$$

Solving the linear regression problem leads to the desired estimate for the relative spatial resolution $\Delta$. In general, if we have several estimates $x \approx x_{i}$ with accuracy $\sigma_{i}$, then, to combine these estimates, we can use the Least Squares Method

$$
\sum_{i} \frac{\left(x-x_{i}\right)^{2}}{\sigma_{i}^{2}} \rightarrow \min
$$

which leads to the estimate

$$
x=\frac{\sum x_{i} \cdot \sigma_{i}^{-2}}{\sum \sigma_{i}^{-2}} .
$$

In our case, we have

$$
\sigma_{i}^{-2}=\frac{\left|F_{2}(\omega)\right|^{2} \cdot\|\omega\|^{4}}{4 n^{2}}
$$

so

$$
\begin{gathered}
\sum x_{i} \cdot \sigma_{i}^{-2}=\frac{1}{4 n^{2}} \cdot \int \frac{\left(\ell_{1}(\omega)-\ell_{2}(\omega)\right)}{\|\omega\|^{2}} \cdot\left|F_{2}(\omega)\right|^{2} \cdot\|\omega\|^{4} d \omega= \\
\frac{1}{4 n^{2}} \cdot \int 2\left(\ell_{1}(\omega)-\ell_{2}(\omega)\right) \cdot\left|F_{2}(\omega)\right|^{2} \cdot\|\omega\|^{2} d \omega
\end{gathered}
$$

and

$$
\sum \sigma_{i}^{-2}=\frac{1}{4 n^{2}} \cdot \int\left|F_{2}(\omega)\right|^{2} \cdot\|\omega\|^{4} d \omega .
$$

We can simplify the resulting expression for the estimate $\Delta$ if we multiply both the numerator and the denominator by the same constant $4 n^{2}$. Thus, we arrive at the following formula for the relative spatial resolution $\Delta$.

\section{Resulting formula for the relative spatial resolution.}

$$
\Delta=2 \frac{\int\left(\ell_{1}(\omega)-\ell_{2}(\omega)\right) \cdot\left|F_{2}(\omega)\right|^{2} \cdot\|\omega\|^{2} d \omega}{\int\left|F_{2}(\omega)\right|^{2} \cdot\|\omega\|^{4} d \omega},
$$

where integration is over frequencies $\omega$ for which

$$
\left|F_{2}(\omega)\right| \geq c \cdot n
$$

for some pre-selected constant $c \gg 1$.

Preliminary results. For simulated 3-D maps, when we artificially introduce different spatial resolutions to the same image, the above method correctly reconstructs the relative spatial resolution.

However, it is difficult to gauge how accurate is our method for the actual geophysical data. The problem is that we do not know the actual density distribution and thus, we do not know for sure how close our estimates are to the actual values of the relative spatial resolution of two geophysical 3-D maps. 


\section{ACKNOWLEDGMENT}

This work was also supported in part by the National Science Foundation grants HRD-0734825 and HRD-1242122 (Cyber-ShARE Center of Excellence) and DUE-0926721.

The authors are thankful to the anonymous referees for valuable suggestions.

\section{REFERENCES}

[1] E. T. Jaynes, Probability Theory: The Logic of Science, Cambridge University Press, Cambridge, Massachisetts, 2003.

[2] G. Klir and B. Yuan, Fuzzy Sets and Fuzzy Logic, Prentice Hall, Upper Saddle River, New Jersey, 1995.

[3] V. Kreinovich, C. Quintana, and L. Reznik. "Gaussian membership functions are most adequate in representing uncertainty in measurements", Proceedings of NAFIPS'92: North American Fuzzy Information Processing Society Conference, Puerto Vallarta, Mexico, December 1517, 1992, NASA Johnson Space Center, Houston, TX, 1992, pp. 618625.

[4] H. T. Nguyen and E. A. Walker, A First Course in Fuzzy Logic, Chapman and Hall/CRC, Boca Raton, Florida, 2006.

[5] O. Ochoa, M. Ceberio, and V. Kreinovich, "How to Describe Spatial Resolution: An Approach Similar to the Central Limit Theorem", Applied Mathematical Sciences, 2010, Vol. 4, No. 63, pp. 3153-3160.

[6] O. Ochoa, A. Velasco, and V. Kreinovich, "Fusing Continuous and Discrete Data, on the Example of Merging Seismic and Gravity Models in Geophysics", Proceedings of the 30th Annual Conference of the North American Fuzzy Information Processing Society NAFIPS'2011, El Paso, Texas, March 18-20, 2011.
[7] O. Ochoa, A. Velasco, and C. Servin, "Towards a Fast, Practical Alternative to Joint Inversion of Multiple Datasets: Model Fusion", Journal of Uncertain Systems, 2011, Vol. 5, No. 2, pp. 119-136

[8] O. Ochoa, A. Velasco, and C. Servin, "Towards Model Fusion in Geophysics: How to Estimate Accuracy of Different Models", Journal of Uncertain Systems, 2013, Vol. 7, No. 3, pp. 190-197.

[9] O. Ochoa, A. A. Velasco, C. Servin, and V. Kreinovich, "Model Fusion under Probabilistic and Interval Uncertainty, with Application to Earth Sciences", In: M. Beer, R. L. Muhanna, and R. L. Mullen (Eds.), Proceedings of the 4th International Workshop on Reliable Engineering Computing REC'2010, Singapore, March 3-5, 2010, pp. 81-100.

[10] O. Ochoa, A. A. Velasco, C. Servin, and V. Kreinovich, "Model Fusion under Probabilistic and Interval Uncertainty, with Application to Earth Sciences", International Journal of Reliability and Safety, 2012, Vol. 6, No. 1-3, pp. 167-187.

[11] C. Servin, O. Ochoa, and A. A. Velasco, "Probabilistic and Interval Uncertainty of the Results of Data Fusion, With Application to Geosciences", Abstracts of 13th International Symposium on Scientific Computing, Computer Arithmetic, and Verified Numerical Computations SCAN'2008, El Paso, Texas, September 29 - October 3, 2008, p. 128.

[12] D. J. Sheskin, Handbook of Parametric and Nonparametric Statistical Procedures, Chapman \& Hall/CRC, Boca Raton, Florida, 2011.

[13] L. A. Zadeh, "Fuzzy sets", Information and Control, 1965, Vol. 8, pp. $338-353$ 